\title{
Long-term spatial tracking of cells affected by environmental insults
}

\author{
Shahid Mohammad ${ }^{1 \dagger}$, Stephen J. Page ${ }^{1 \dagger}$, Toru Sasaki ${ }^{1,2}$, Nicholas Ayvazian ${ }^{1,3}$, Pasko Rakic ${ }^{4}$, \\ Yuka Imamura Kawasawa ${ }^{5,6}$, Kazue Hashimoto-Torii ${ }^{1,7^{*}}$ and Masaaki Torii ${ }^{1,7^{*}}$
}

\begin{abstract}
Background: Harsh environments surrounding fetuses and children can induce cellular damage in the developing brain, increasing the risk of intellectual disability and other neurodevelopmental disorders such as schizophrenia. However, the mechanisms by which early damage leads to disease manifestation in later life remain largely unknown. Previously, we demonstrated that the activation of heat shock (HS) signaling can be utilized as a unique reporter to label the cells that undergo specific molecular/cellular changes upon exposure to environmental insults throughout the body. Since the activation of HS signaling is an acute and transient event, this approach was not intended for long-term tracing of affected cells after the activation has diminished. In the present study, we generated new reporter transgenic mouse lines as a novel tool to achieve systemic and long-term tracking of affected cells and their progeny.
\end{abstract}

Methods: The reporter transgenic mouse system was designed so that the activation of HS signaling through HS response element (HSE) drives flippase (FLPO)-flippase recognition target (FRT) recombination-mediated permanent expression of the red fluorescent protein (RFP), tdTomato. With a priority on consistent and efficient assessment of the reporter system, we focused on intraperitoneal (i.p.) injection models of high-dose, short prenatal exposure to alcohol (ethanol) and sodium arsenite (ethanol at $4.0 \mathrm{~g} / \mathrm{kg} /$ day and sodium arsenite at $5.0 \mathrm{mg} / \mathrm{kg} /$ day, at embryonic day (E) 12 and 13). Long-term reporter expression was examined in the brain of reporter mice that were prenatally exposed to these insults. Electrophysiological properties were compared between RFP ${ }^{+}$and RFP $^{-}$cortical neurons in animals prenatally exposed to arsenite.

Results: We detected RFP ${ }^{+}$neurons and glia in the brains of postnatal mice that had been prenatally exposed to alcohol or sodium arsenite. In animals prenatally exposed to sodium arsenite, we also detected reduced excitability in $\mathrm{RFP}^{+}$cortical neurons.

Conclusion: The reporter transgenic mice allowed us to trace the cells that once responded to prenatal environmental stress and the progeny derived from these cells long after the exposure in postnatal animals. Tracing of these cells indicates that the impact of prenatal exposure on neural progenitor cells can lead to functional abnormalities in their progeny cells in the postnatal brain. Further studies using more clinically relevant exposure models are warranted to explore this mechanism.

Keywords: Environmental stress, Brain development, Heat shock signaling, Reporter, Lineage tracing

\footnotetext{
* Correspondence: KHTorii@cnmc.org; MTorii@childrensnational.org

†'Shahid Mohammad and Stephen J. Page contributed equally to this work.

'Center for Neuroscience Research, Children's Research Institute, Children's

National Hospital, Washington, DC, USA

Full list of author information is available at the end of the article
}

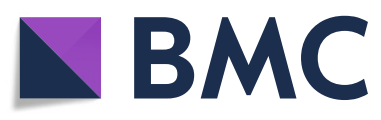

(c) The Author(s). 2020 Open Access This article is licensed under a Creative Commons Attribution 4.0 International License, which permits use, sharing, adaptation, distribution and reproduction in any medium or format, as long as you give appropriate credit to the original author(s) and the source, provide a link to the Creative Commons licence, and indicate if changes were made. The images or other third party material in this article are included in the article's Creative Commons licence, unless indicated otherwise in a credit line to the material. If material is not included in the article's Creative Commons licence and your intended use is not permitted by statutory regulation or exceeds the permitted use, you will need to obtain permission directly from the copyright holder. To view a copy of this licence, visit http://creativecommons.org/licenses/by/4.0/. The Creative Commons Public Domain Dedication waiver (http://creativecommons.org/publicdomain/zero/1.0/) applies to the data made available in this article, unless otherwise stated in a credit line to the data. 


\section{Background}

Exposure to environmental insults such as chemicals or infectious agents during development alters the molecular properties of afflicted cells [1], affecting various critical cellular processes including those in brain development [2-6], and increasing the risks of neuropsychiatric disorders [7]. However, the mechanisms by which environmental impact during the early developmental period leads to variable disease manifestation in later life remain largely unknown.

Activation of HS signaling, which is not limited by heat stress alone but by various types of environmental stress, is one of the intrinsic cytoprotective mechanisms known to protect the developing brain from deleterious outcomes [8-10]. We previously generated reporter transgenic mice, in which heat shock factor 1 (Hsf1)-mediated acute activation of HS signaling is visualized by the expression of RFP reporter under the control of the HS response element (HSE) that contains the mouse $H s p 70$ promoter $[11,12]$. Using these mice, we have shown that the acute activation of HS signaling in the embryonic brain upon exposure to environmental insults, such as alcohol and sodium arsenite, is heterogeneous among neural progenitor cells and occurs in a stochastic manner $[11,12]$. We have also demonstrated that cells labeled by reporter expression exhibit deficits in cell cycling and migration during embryonic cortical development [11, 12], indicating that the cells which underwent HS signaling activation and survived the exposure may contribute to brain malfunction in later life. It has also been shown that HS signaling can mark epigenetic transcriptional memory, and thereby causes long-term changes in gene expression [13]. These lines of evidence prompt the need for a novel tool that can track these affected cells and their progeny, and address the mechanism underlying long-term consequences of prior impacts. The reporter transgenic mice that we previously generated $[11,12]$, however, were not specifically designed for such a tracing purpose, and only retain reporter expression for a few days after stress exposure.

In the present study, we report the generation of novel reporter transgenic mice that harbor a combination of the HSE-driven FLPo and FRT-RFP transgenes for longterm lineage tracing of cells in which HS signaling was activated. This novel animal model allowed us to explore the impact of prenatal alcohol or arsenic exposure on neuronal functions in the cerebral cortex of postnatal animals.

\section{Methods}

\section{Animals and drug administration}

All mice were maintained on a light-dark cycle (lights on 06:00-18:00 h) at a constant temperature $\left(22 \pm 1^{\circ} \mathrm{C}\right)$. Pregnant mice received i.p. injections of $25 \%$ ethanol in
PBS at $4.0 \mathrm{~g} / \mathrm{kg} /$ day or sodium arsenite in PBS at $5.0 \mathrm{mg} /$ $\mathrm{kg} /$ day at E12 and E13. PBS alone was injected as the control for immunohistochemistry experiments. Untreated animals were used as controls for electrophysiology experiments. Weights of carcasses, brains, and lungs were measured at necropsy. All protocols were approved by the Institutional Animal Care and Use Committee (IACUC) of the Children's National Hospital. All methods were performed in accordance with relevant guidelines and regulations.

\section{Generation of the HSE-FLPo mouse}

Using Vista Genome Browser (pipeline.lbl.gov/cgi-bin/ gateway2), the oligonucleotide including HSE [12] and codon-optimized flippase (FLPo) [14] sequence was synthesized and cloned into pUC57 (Genscript). Kpn1 and Sma1 recognition sites were added at the ends of the following 2252-bp sequence for synthesis.

HSE-FLPo:

CAGCTTCACCCACAGGGACCCCGAAGTTGCGTCG CCTCCGCAACAGTGTCAATAGCAGCACCAGCACT TCCCCACACCCTCCCCCTCAGGAATCCGTACTCT CTAGCGAACCCCAGAAACCTCTGGAGAGTTCTGG ACAAGGGCGGAACCCACAACTCCGATTACTCAAG GGAGGCGGGGAAGCTCCACCAGACGCGAAACTG CTGGAAGATTCCTGGCCCCAAGGCCTCCTCCGGCT CGCTGATTGGCCCAGCGGAGAGTGGGCGGGGCCG GTGAAGACTCCTTAAAGGCGCAGGGCGGCGAG CAGGGCACCAGACGCTGACAGCTACTCAGAATCA AATCTGGTTCCATCCAGAGACAAGCGAAGACAAG AGAAGCAGAGCGAGCGGCGCGTTCCCGATCCTCG GCCAGGACCAGCCTTCCCCAGAGCATCCACGCCG CGGAGCGCAACCTTCCCAGGAGCATCCCTGCCGCG GAGCGCAACTTTCCCCGGAGCATCCACGCCGCG GAGCGCAGCCTTCCAGAAGCAGAGCGCGGCGCCA TGGCCAAGAACACGGCGATCGGCATCGACCTGGG CACCACCTACTCGTGCGTGGGCGTGTTCCAGCA CGGCAAGGTGGAGATCATCGCCAACGACCAGG GCAACCGCCGGTGGCGGCCGCGATCAAGCTTCTG CAATCGCCGCCACCATGGCTCCTAAGAAGAAGAG GAAGGTGATGAGCCAGTTCGACATCCTGTGCAAG ACCCCCCCCAAGGTGCTGGTGCGGCAGTTCGTGG AGAGATTCGAGAGGCCCAGCGGCGAGAAGATCGC CAGCTGTGCCGCCGAGCTGACCTACCTGTGCT GGATGATCACCCACAACGGCACCGCCATCAAG AGGGCCACCTTCATGAGCTACAACACCATCATCAG CAACAGCCTGAGCTTCGACATCGTGAACAAGA GCCTGCAGTTCAAGTACAAGACCCAGAAGGCC ACCATCCTGGAGGCCAGCCTGAAGAAGCTGATCCC CGCCTGGGAGTTCACCATCATCCCTTACAACGGCC AGAAGCACCAGAGCGACATCACCGACATCGTGTC CAGCCTGCAGCTGCAGTTCGAGAGCAGCGAGGA GGCCGACAAGGGCAACAGCCACAGCAAGAAGATG CTGAAGGCCCTGCTGTCCGAGGGCGAGAGCATCT 
GGGAGATCACCGAGAAGATCCTGAACAGCTTCGA GTACACCAGCAGGTTCACCAAGACCAAGACCCTG TACCAGTTCCTGTTCCTGGCCACATTCATCAACTG CGGCAGGTTCAGCGACATCAAGAACGTGGACCCC AAGAGCTTCAAGCTGGTGCAGAACAAGTACCTGG GCGTGATCATTCAGTGCCTGGTGACCGAGACCAA GACAAGCGTGTCCAGGCACATCTACTTTTTCAGC GCCAGAGGCAGGATCGACCCCCTGGTGTACCT GGACGAGTTCCTGAGGAACAGCGAGCCCGTGCTG AAGAGAGTGAACAGGACCGGCAACAGCAGCAG CAACAAGCAGGAGTACCAGCTGCTGAAGGACA ACCTGGTGCGCAGCTACAACAAGGCCCTGAAGAA GAACGCCCCCTACCCCATCTTCGCTATCAAGAAC GGCCCTAAGAGCCACATCGGCAGGCACCTGATG ACCAGCTTTCTGAGCATGAAGGGCCTGACCGAGC TGACAAACGTGGTGGGCAACTGGAGCGACAAGA GGGCCTCCGCCGTGGCCAGGACCACCTACACCCA CCAGATCACCGCCATCCCCGACCACTACTTCGCCC TGGTGTCCAGGTACTACGCCTACGACCCCATCAG CAAGGAGATGATCGCCCTGAAGGACGAGACCAAC CCCATCGAGGAGTGGCAGCACATCGAGCAGCTGA AGGGCAGCGCCGAGGGCAGCATCAGATACCCCGC CTGGAACGGCATCATCAGCCAGGAGGTGCTGGAC TACCTGAGCAGCTACATCAACAGGCGGATCTGAT GAGATATCCTAGAGCTCGCTGATCAGCCTCGACT GTGCCTTCTAGTTGCCAGCCATCTGTTGTTTGC CCCTCCCCCGTGCCTTCCTTGACCCTGGAAGGTG CCACTCCCACTGTCCTTTCCTAATAAAATGAGGA AATTGCATCGCATTGTCTGAGTAGGTGTCATTCT ATTCTGGGGGGTGGGGTGGGGCAGGACAGCAAG
GGGGAGGATTGGGAAGACAATAGCAGGCATGCT GGGGATGCGGTGGGCTCTATGGCTTCTG.

To generate HSE-FLPo transgenic mice, the HSE-FLPo fragment (2301 bp) was excised using KpnI, SmaI, and ScaI and purified according to the standard protocol for microinjection. Prior to the injection, the 2301-bp band was further verified with the expected 1216- and 1085bp products of AhdI digestion (Fig. 1b). Microinjection into the C57BL/6 J X C57BL/6 J strain was performed by Cyagen. Eleven out of 63 founder lines were screened by PCR genotyping for FLPo. The tails of the 11 pups were re-cut and the insertion of transgene was confirmed by PCR (Fig. S1) using the following primers: transgene primers F1: AGATCATCGCCAACGACCAG and R1: TAAGGGATGATGGTGAACTCCCAG (product size: $393 \mathrm{bp}$ ); internal control primers F1: CTATCAGGGA TACTCCTCTTTGCC and R1: GATACAGGAATGACAAGCTCATGGT (product size: $507 \mathrm{bp}$ ); transgene primers F2: AGGCACCTGATGACCAGCTTTC and R2: ACAACAGATGGCTGGCAACTAG (product size: $415 \mathrm{bp}$ ); internal control primers F2: GCAGAAGAGG ACAGATACATTCAT and R2: CCTACTGAAGAATC TATCCCACAG (product size: $689 \mathrm{bp}$ ). The internal control PCR targets the endogenous mouse Rgs7 (G protein signaling 7) locus. PCR was carried out in $25 \mu \mathrm{L}$ volume for 38 cycles with the annealing temperature at $60^{\circ} \mathrm{C}$ under standard conditions, with all four primers listed above added to each reaction. Taq DNA polymerase used was TaKaRa R004A. Three controls used in PCR genotyping were (a) water control: no DNA

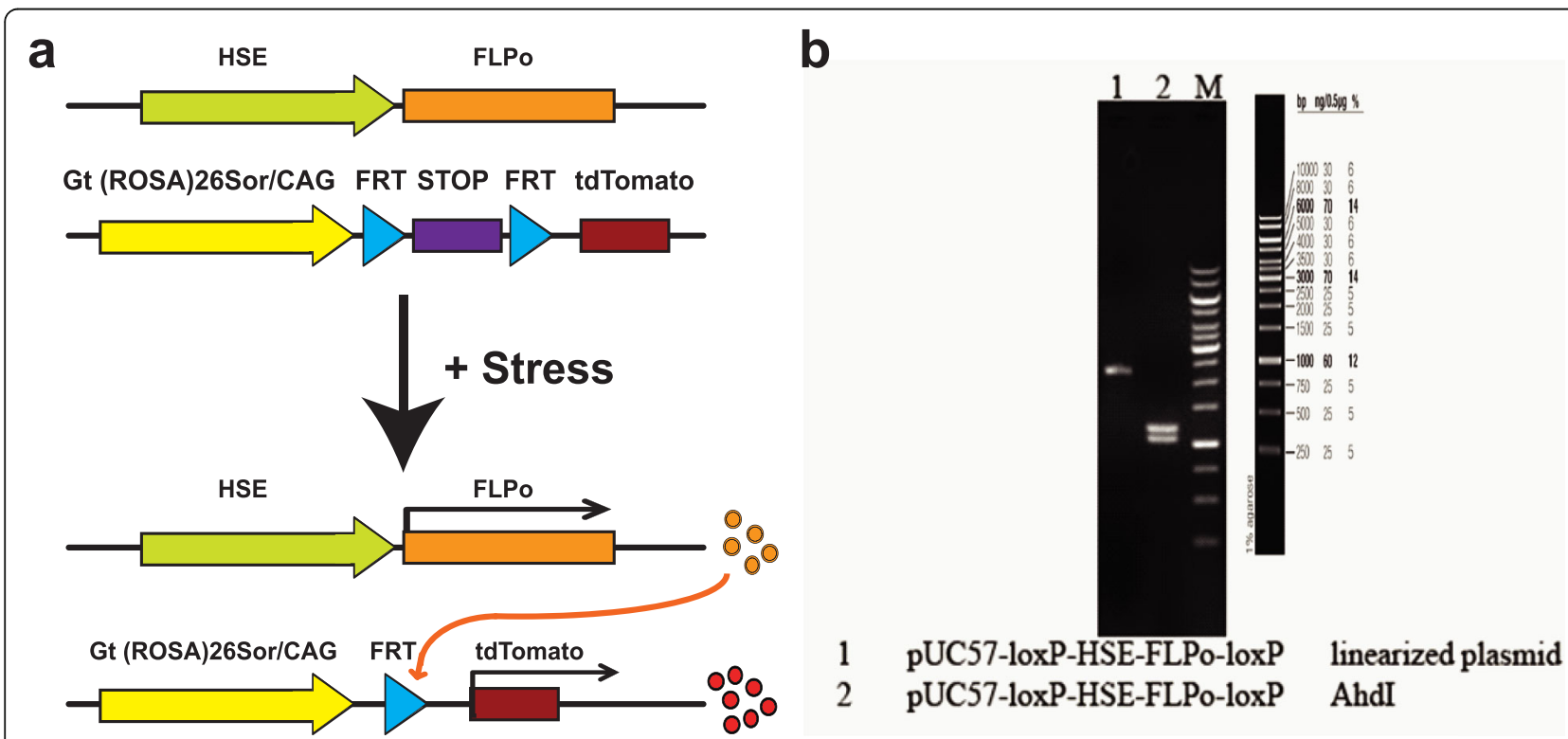

Fig. 1 The reporter construct for long-term detection of cell lineages that experienced HSF1 activation. a The design of RFP reporter system, in which the HSE and FLPO-FRT recombination system are combined for long-term labeling. Upon exposure to stress, HSE drives the expression of FLPo, which then induces RFP expression via FRT recombination. $\mathbf{b}$ The HSE-FLPo fragment (2301 bp) was verified by Ahdl digestion that generated the expected 1216 and 1085 bp products 
template added, (b) positive control: $400 \mathrm{ng}$ of mouse genomic DNA spiked with an amount of transgene injection DNA that was equivalent to 5 copies of transgene per diploid mouse genome, and (c) wildtype control: $400 \mathrm{ng}$ of mouse genomic DNA.

The 11 founder lines (A1-A11) were then separately crossed with RC::FLTG mice [B6.Cg-Gt(ROSA)26Sortm1.3(CAG-tdTomato,-EGFP)Pjen/J, Stock No: 026932, The Jackson Laboratory], in which FLPo-FRT recombination results in high expression of tdTomato, and F1 generations were exposed to ethanol or sodium arsenite at E12 and E13 to confirm the reporter expression. One of the three founder lines that showed the most consistent expression of reporter was further examined in this study. The routine genotyping of double transgenic animals was performed by Transnetyx.

Whole-genome sequencing of HSE-RFP transgenic mouse Shallow whole-genome sequencing of the genomic DNA extracted from transgenic mice was performed using an Illumina NovaSeq 6000 at $\sim 151$-bp paired-end aiming at $\sim 3 \times$ whole-genome coverage [15]. To identify candidate integration sites, paired-end reads were aligned to the reference mouse genome using Bowtie2 [16] in global (reads align end to end) and local (reads are softclipped) modes allowing 0 or 1 mismatches for potential SNPs or PCR errors [17, 18], and discordant read pairs were reported. As an alternative approach, we also ran Burrows-Wheeler Aligner (BWA) using the BWA-MEM (maximum exact matches), which performs local alignment and supports split (chimeric) read alignments [18, 19]. To calculate the depth of sequencing coverage, we used GATK DepthofCoverage tool with the mouse reference genome (GRCm38).

\section{Validation of transgene insertion loci by polymerase chain reaction}

To validate the transgene insertion in chromosomes 18 and 19 , polymerase chain reaction was performed using the following pairs of primers designed within the FLPo sequence or intronic regions flanking the insertion site: FLPoF: TGATGAGCCAGTTCGACATC and chr18R: CCCTGCCAGAGACAGACAAC (for chromosome 18), chr19F: TGAGCCTTTCTGGCTTCAGG and chr19R: TGAGCCTTTCTGGCTTCAGG (for chromosome 19). Genomic DNA was extracted from the brain or tail tissue using MinElute PCR Purification Kit (Qiagen). PCR was performed using Takara PCR master mixes (chromosome 18) or Promega GoTaq (chromosome 19) with 35 cycles at annealing temperature of $55^{\circ} \mathrm{C}$. PCR products were detected using the conventional agarose gel electrophoresis method or BioAnalyzer (Agilent Technologies).

\section{Immunohistochemistry}

Mice were perfused with $4 \%$ paraformaldehyde (PFA) following a standard protocol. The brains were collected and post-fixed in $4 \%$ PFA at $4{ }^{\circ} \mathrm{C}$ overnight, followed by incubation in $10 \%$ and $30 \%$ sucrose in PBS at $4{ }^{\circ} \mathrm{C}$ for $24 \mathrm{~h}$. Coronal sections $(60 \mu \mathrm{m}$ thickness $)$ were made using a cryostat (CM3050S; Leica). Free-floating sections were treated by hydrogen peroxide in methanol (1:4) solution at $-20^{\circ} \mathrm{C}$ for $20 \mathrm{~min}$ to inactivate endogenous peroxidase activity. After washing with PBS-T (PBS containing $0.2 \%$ Tween 20 ) three times, sections were incubated with blocking buffer [ $2 \%$ bovine serum albumin (BSA) in PBS-T] for $30 \mathrm{~min}$ at room temperature. Sections were then incubated with a rabbit anti-RFP antibody diluted in the blocking buffer (1:500) (Abcam) overnight at $4{ }^{\circ} \mathrm{C}$. Incubation with a horseradish peroxidase (HRP)-conjugated anti-rabbit IgG (1:500) (Jackson ImmunoResearch) was for $3 \mathrm{~h}$ at room temperature. TSA kit (PerkinElmer) was used for signal amplification. DAPI (4',6-diamidino-2-phenylindole, dihydrochloride) $(1: 10,000)$ (Sigma-Aldrich) was used for nuclear counterstaining. Labeled sections were imaged using an Olympus confocal microscope equipped with a digital camera.

\section{Coronal slice preparation for electrophysiology}

Reporter transgenic mice (P15-21) were used for electrophysiology experiments. No significant differences were observed on the basis of sex, and therefore data collected from males and females were combined for analysis. Mice were anesthetized by inhalation of isoflurane $(2-4 \%)$ and decapitated. The forebrain was removed and placed for $1 \mathrm{~min}$ in cold $\left(0-4{ }^{\circ} \mathrm{C}\right)$ artificial cerebrospinal fluid (aCSF) composed of the following (in $\mathrm{mM}$ ): $\mathrm{NaCl} 125, \mathrm{KCl} 3, \mathrm{KH}_{2} \mathrm{PO}_{4} 1.2, \mathrm{MgSO}_{4} 1.2, \mathrm{NaHCO}_{3} 25$, dextrose $10, \mathrm{CaCl}_{2}$ 2, and bubbled with $95 \% \mathrm{O}_{2} / 5 \% \mathrm{CO}_{2}$ ( $\mathrm{pH}$ 7.4, 306-310 mOsM adjusted with dextrose). Using a vibrating microtome (Leica VT-1000S), 300- $\mu$ m-thick coronal forebrain slices were cut. Slices were incubated at room temperature in a submersion chamber containing an identical oxygenated aCSF for at least 45 mins before use.

\section{Electrophysiology}

Cortical neurons expressing RFP were visually selected for electrophysiological patch-clamp recordings using a fluorescence microscope (Olympus BX51WI). Patch electrodes were guided to neurons using differential interference contrast (DIC) optics illuminated with infrared light. Voltage-clamp and current-clamp recordings were made with a MultiClamp $700 \mathrm{~B}$ amplifier, Digidata 1440A digitizer, and pClamp10.7 software (all from Molecular Devices). Data were filtered at $10 \mathrm{kHz}$ and stored on a personal computer for offline analysis. For 
recordings, patch pipettes were pulled using a horizontal pipette puller (P-2000, Sutter Instruments). The pipette resistance was 6.0-9.0 $\mathrm{M} \Omega$ when filled with internal solution (in $\mathrm{mM}: \mathrm{NaCl} \mathrm{10,} \mathrm{K-gluconate} \mathrm{130,} \mathrm{EGTA} \mathrm{11,}$ $\mathrm{CaCl}_{2} 1, \mathrm{MgCl}_{2}$ 2, HEPES 10, Na-ATP 2, Na-GTP 0.2, $\mathrm{pH}$ 7.3, 297-300 mOsM). After formation of a stable gigaohm seal $(>1 \mathrm{G} \Omega)$, the whole-cell configuration was established. Only neurons with holding currents not exceeding $\pm 200 \mathrm{pA}$ at $V_{\text {Hold }}=-60 \mathrm{mV}$ for the 10 -min control period were studied further. Access resistance (Ra) was monitored throughout recordings, and neurons were not included in additional analysis if it exceeded $10 \mathrm{M} \Omega$ or drifted $>20 \%$. Whole-cell recordings were made in extracellular solution that was identical to oxygenated aCSF used for brain tissue preparation. aCSF was heated to $32 \pm 0.5^{\circ} \mathrm{C}$ using a pre-heater, temperature sensor (positioned on the wall of the recording chamber next to the slice), and temperature controller (Warner Instruments).

\section{Data analysis}

All values are reported as mean \pm SEM unless otherwise indicated. In box plots, the line within the box indicates the median, and the upper and lower edges of the box represent the 25th and 75th percentiles, respectively. The upper and lower whisker boundaries indicate the 10th and 90th percentiles, respectively. All quantitative data were statistically analyzed using chi-square test or one-way analysis of variance (ANOVA) (with repeated measures where appropriate), using GraphPad Prism version 7 (GraphPad Software) or Origin 9 software (OriginLab). $P<0.05$ values were considered statistically significant.

\section{Results}

Generation of reporter transgenic mice for long-term labeling of cell lineages in which heat shock signaling is activated

To generate reporter transgenic mice for in vivo longterm tracking of cell lineages that responded to environmental insults via activation of HS signaling, we designed a reporter system in which the activation of HS signaling drives the FLPo-FRT recombination-mediated expression of RFP (Fig. 1). A similar reporter system has been validated in vivo using in utero electroporationmediated gene transfer method in our previous studies $[12,20]$. For the 11 founder lines we obtained, we confirmed the insertion of the HSE-FLPo transgene by PCR (Fig. S1). After crossing these HSE-FLPo transgenic mice with $R C:: F L T G$ mice, in which RFP (tdTomato) expression is triggered by FLPo-FRT recombination, we examined RFP expression in the brains of adolescent mice (at P20) born from dams that had been administered alcohol $(4.0 \mathrm{~g} / \mathrm{kg} /$ day $)$ during embryonic days $(\mathrm{E})$
12 and 13. Of the three HSE-FLPo founder lines (lines A4, A6, and A8), in which we observed strong expression of RFP in the brain, one line (A4) was further analyzed. Shallow whole-genome sequencing was performed to define the loci of transgenes in the F2 generation. $95 \%$ of the genome was covered at least $1 \times$, and the mean mapped read depth was $3.54 \times$ with an interquartile range of 3 , suggesting enough coverage to detect chromosomal structural variations [21]. The sequencing confirmed that the genomic loci of inserted transgene were located in intronic regions in the direction opposite to DNA transcription (Figs. S2, S3), suggesting a low possibility of disturbance in intrinsic gene expression in the reporter mice. After several generations of breeding (after the F5 generation), polymerase chain reaction (PCR) was performed to further validate the transgene insertion. The results indicated that these mice inherited and maintained the transgene only in chromosome 18 in a stable manner (Figs. S2, S3). All the following studies were performed using this mouse line (A4) that maintains the transgene only in chromosome 18.

\section{Tracking of the cell lineages affected by prenatal exposure to environmental insults in the postnatal brain}

To test whether the new reporter mice allow long-term tracing of cell lineages affected by environmental insults, we used models of prenatal exposure to alcohol and arsenic as two representative environmental insults and examined the consequence postnatally. Prenatal alcohol (ethanol) exposure due to maternal consumption of alcohol during pregnancy causes a broad range of adverse developmental effects, including physical, behavioral, and learning problems that are collectively referred to as fetal alcohol spectrum disorders (FASD) [22, 23]. Arsenite is an inorganic form of arsenic, a metalloid naturally found in soil, air, and water in some zones of the world. Cumulative evidence from epidemiological and animal studies indicates that arsenic exposure in utero or in early childhood causes developmental neurotoxicity, affecting intellectual functions throughout life [24-29].

For efficient assessment of the reporter system, we focused on high-dose, short exposure models via i.p. injections; pregnant transgenic mice were administered ethanol $(4.0 \mathrm{~g} / \mathrm{kg} /$ day $)$ or sodium arsenite $(5.0 \mathrm{mg} / \mathrm{kg} /$ day) (or PBS as control) at E12 and E13. With these conditions, no obvious toxicity was observed in dams and pups. Body weight and major organ weights were not affected in the pups at P20 (Fig. S4). In both ethanol- and arsenite-exposed pups, but not in PBS-exposed pups, we observed RFP expression in neurons and glial cells scattered across various brain regions, including the cerebral cortex, hippocampus, striatum, thalamus, and hypothalamus (Figs. 2 and 3). The expression patterns were different between individual animals (Figs. 2 and 3), 


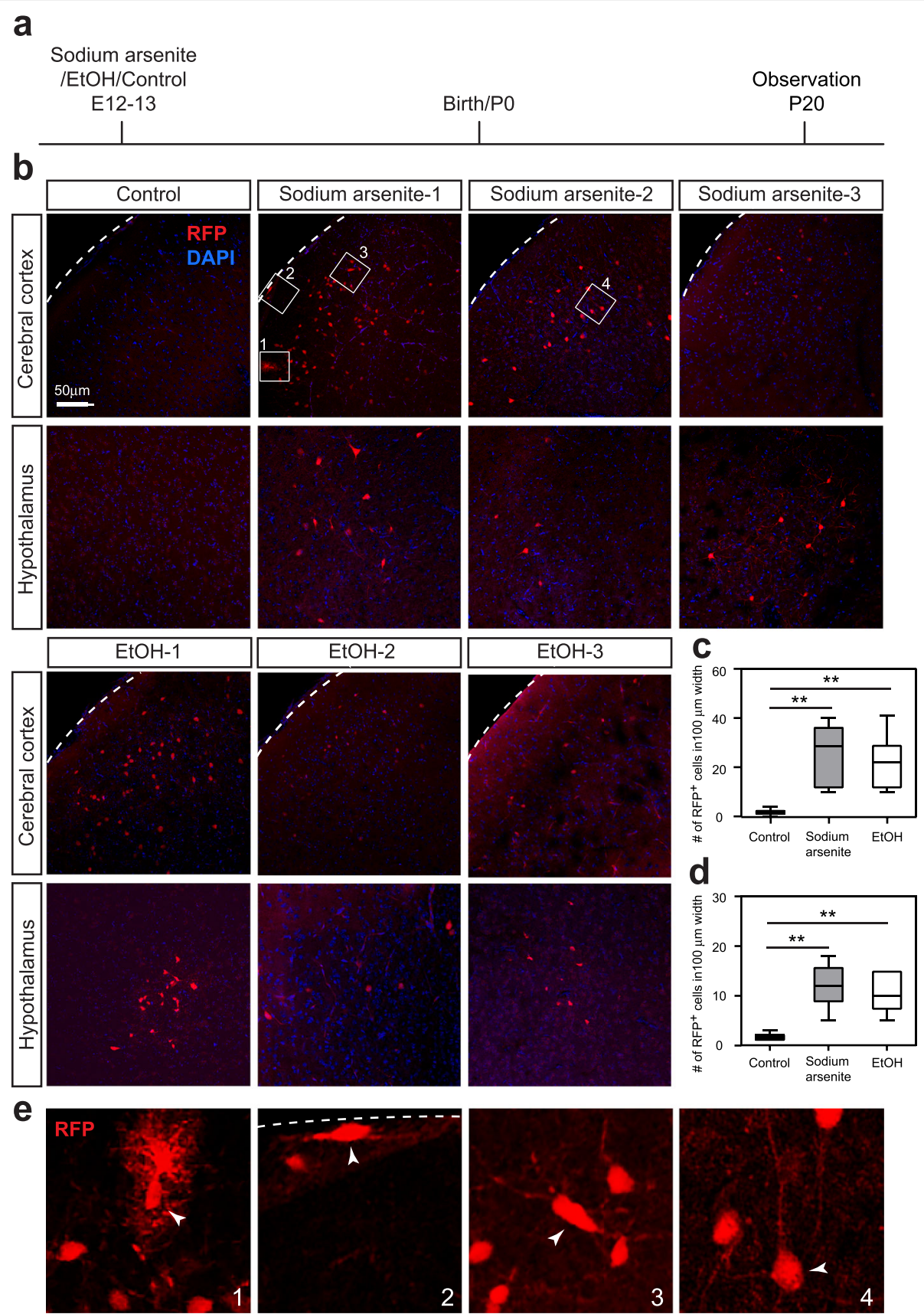

Fig. 2 RFP reporter expression in the postnatal brain of reporter transgenic mice prenatally exposed to sodium arsenite or ethanol. a Experimental timeline. $\mathbf{b}$ Immunohistochemistry for RFP in the cerebral cortex and hypothalamus of the reporter transgenic mice (line A4) that were prenatally exposed to PBS (control), sodium arsenite, or ethanol (EtOH) as indicated. Results from 3 different animals each are shown for sodium arsenite- and ethanol-exposed groups. Broken lines indicate the pial surface. Sections were counterstained with DAPI. Observations were made at P20. $\mathbf{c}$ The average density of RFP ${ }^{+}$cells (in coronal sections) throughout the cerebral cortex; $F(2,15)=10.66, P=0.001$ by one-way ANOVA, ${ }^{* *} P<0.01$ by Tukey test. $\mathbf{d}$ The average density of RFP ${ }^{+}$cells (in coronal sections) throughout the hypothalamus; $F(2,15)=15.69, P=$ 0.0002 by one-way ANOVA, ${ }^{* *} P<0.01$ by Tukey test. e Higher magnification views of the boxed areas $1-4$ in (b). Pial surface is oriented to the top. Reporter expression was observed in various cell types in the cerebral cortex, including glial (1), presumptive Cajal-Retzius cell (2), interneuron (3), and pyramidal neuron (4) (arrowheads). The broken line indicates the pial surface 


\section{Sodium arsenite}
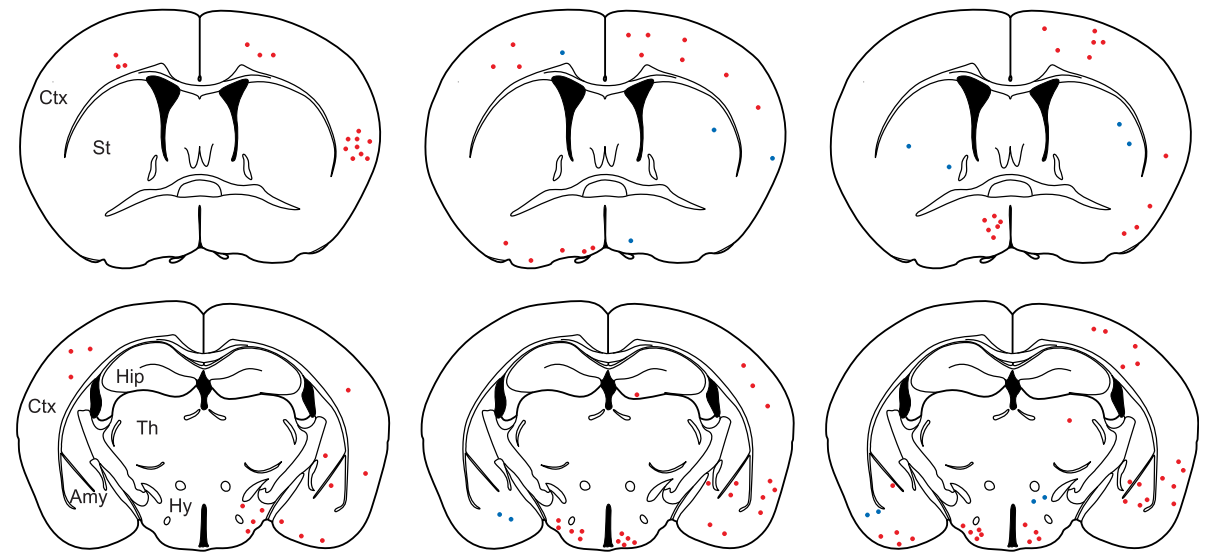

\section{$\mathrm{EtOH}$}
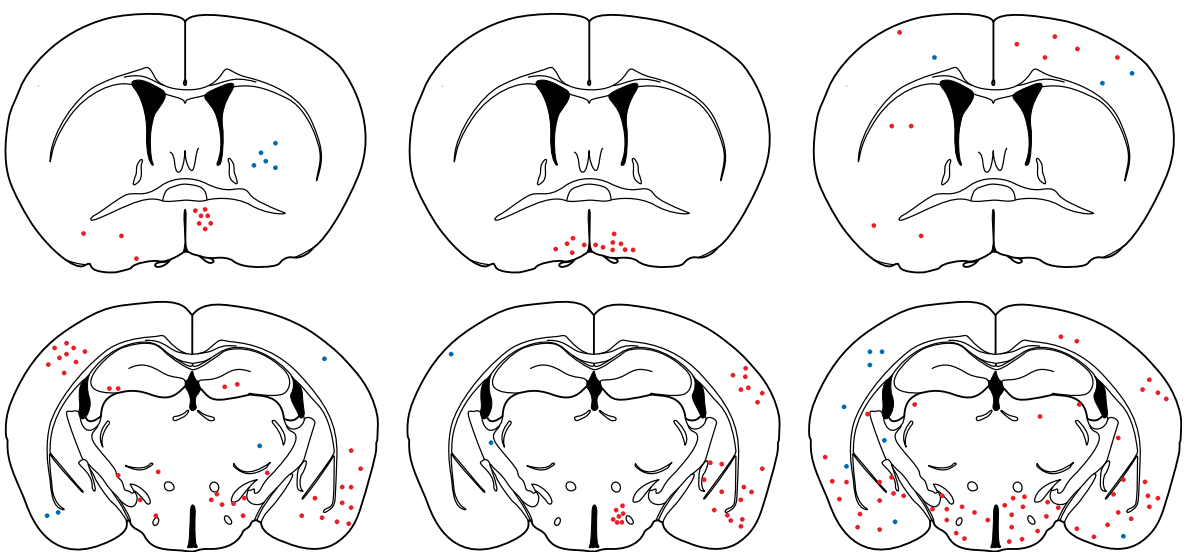

Fig. 3 Schematic representation of the distribution of RFP-positive cells in the anterior and posterior parts of the forebrain in sodium arseniteand ethanol-exposed mice. Results from three P20 mice each are shown. Red dots and blue dots indicate, neurons and glial cells, respectively. Each dot represents 2-3 cells. Ctx: cerebral cortex; Hip: hippocampus; St: striatum; Th: thalamus; Hy: hypothalamus; Amy: amygdala

consistent with the stochastic pattern of HS signaling activation by exposure to environmental insults in neural progenitor cells in the embryonic brain $[10,12,30]$.

\section{Abnormal electrophysiological properties of reporter- positive neurons in the brain of postnatal animals that were prenatally exposed to environmental insults}

To test whether the impact of prenatal exposure, marked by the activation of HS signaling in neural progenitors, alters electrophysiological properties in their progeny after birth, embryos of reporter transgenic mice were exposed to sodium arsenite during E12 and E13 by i.p. injecting pregnant dams with sodium arsenite $(5.0 \mathrm{mg}$ / $\mathrm{kg}$ /day). Offspring were sacrificed for acute brain slice whole-cell recording from cortical pyramidal neurons in the primary motor cortex (layers II and III) at P15-21 (Fig. 4a). Neurons negative for RFP expression, from sodium arsenite-treated or untreated mice were recorded as controls. Voltage-clamp recordings revealed no overall differences in neuronal membrane properties between $\mathrm{RFP}^{+}$and $\mathrm{RFP}^{-}$pyramidal neurons (Fig. 4b). Additionally, recordings performed at $-60 \mathrm{mV}$ to evaluate spontaneous excitatory post-synaptic currents (sEPSC) showed no differences between any of the three groups of neurons (Fig. $4 \mathrm{c}$ and $\mathrm{d}$ ), indicating that fastexcitatory synaptic transmission was not altered by prenatal exposure to sodium arsenite.

To investigate whether action potential (AP) firing is disrupted by sodium arsenite exposure during pregnancy, we performed current-clamp recordings in $\mathrm{RFP}^{+}$ and $\mathrm{RFP}^{-}$neurons from reporter transgenic mice that were exposed to sodium arsenite prenatally, as well as in $\mathrm{RFP}^{-}$neurons from untreated reporter transgenic mice. Recorded neurons were maintained at a holding potential of $-60 \mathrm{mV}$, and a series of current steps $(-100 \mathrm{pA}$ to $395 \mathrm{pA}, 500 \mathrm{~ms}, 5 \mathrm{pA}$ steps) were applied to study AP 


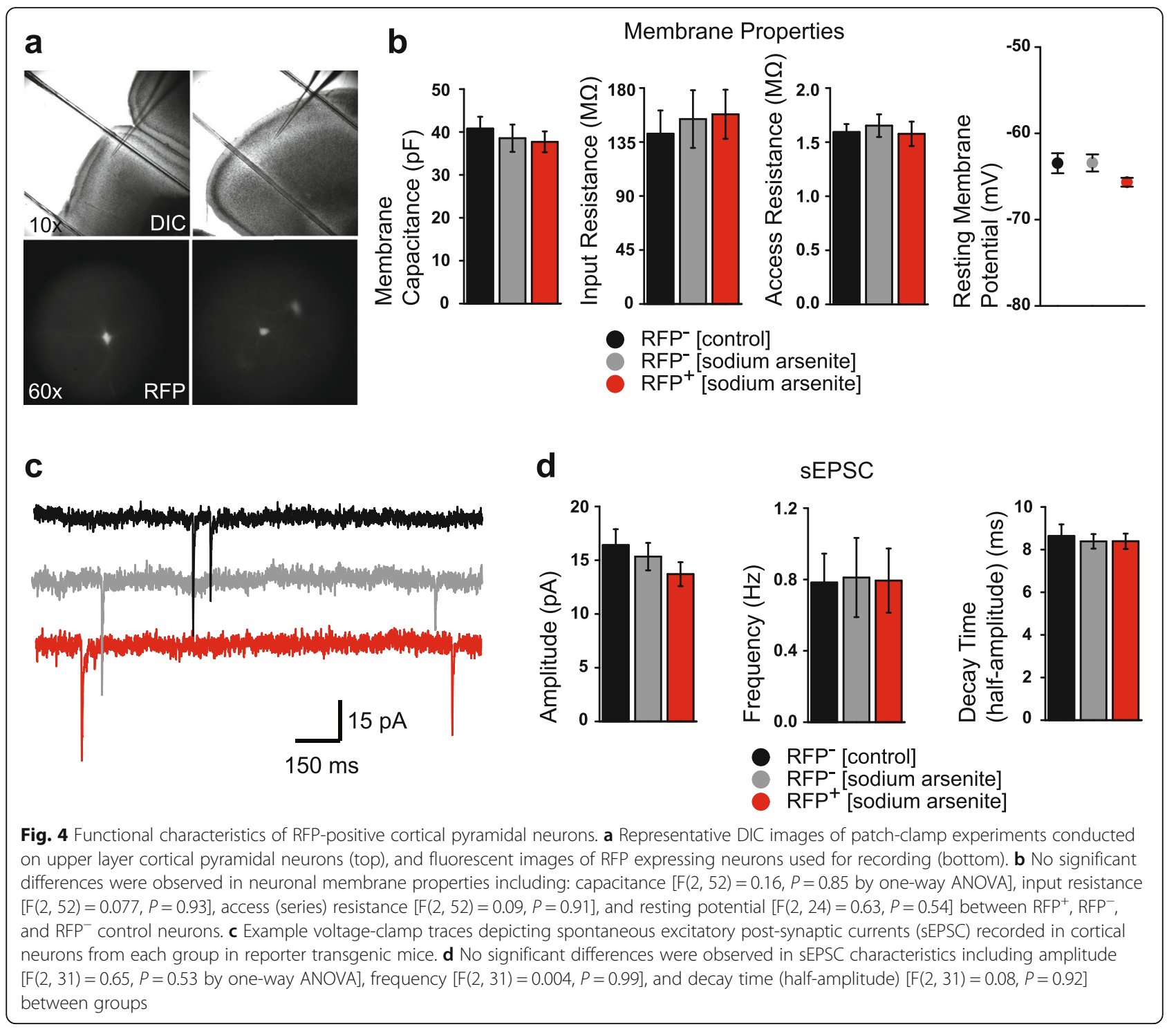

firing across a range of stimulation intensities. It was found that, while $\mathrm{RFP}^{-}$neurons, from both sodium arsenite and untreated groups, tended to follow a typical stimulation-frequency curve, a large portion of $\mathrm{RFP}^{+}$ neurons exhibited mono-spiking, in which a single AP was evoked by a current step at threshold, and for all stimulus intensities above firing threshold (Fig. 5a). Out of all $\mathrm{RFP}^{+}$neurons tested, 50\% (7/14) were classified as mono-spiking neurons, whereas only $15 \%(2 / 13)$ of $\mathrm{RFP}^{-}$ neurons from sodium arsenite treated mice, and none $(0 / 12)$ of the $\mathrm{RFP}^{-}$neurons tested in untreated mice produced single-spikes (Fig. 5b). Among the $\mathrm{RFP}^{+}$neurons that did engage in typical spiking behavior (7/14 polyspiking), no differences were found in average stimulation-frequency curve when compared to $\mathrm{RFP}^{-}$ neurons from either sodium arsenite or untreated groups (Fig. 5c). Finally, it was found that $\mathrm{RFP}^{+}$neurons required a greater amount of current injection to reach the AP firing threshold (Fig. 5d) and produced slightly narrower 1st APs (Fig. $5 \mathrm{f}$ and g) without a change in 1st AP amplitude (Fig. 5e). Together, our results indicate that excitability is generally reduced in RFP+ neurons in the arsenite-exposed cortex, as they are significantly more likely to produce single, narrow spikes at an increased current threshold.

\section{Discussion}

In the present study, we report that our novel transgenic mouse line enables long-term lineage tracing of cells that had once activated HS signaling to respond to environmental insults, using two different prenatal exposure models (alcohol and arsenic exposure). 

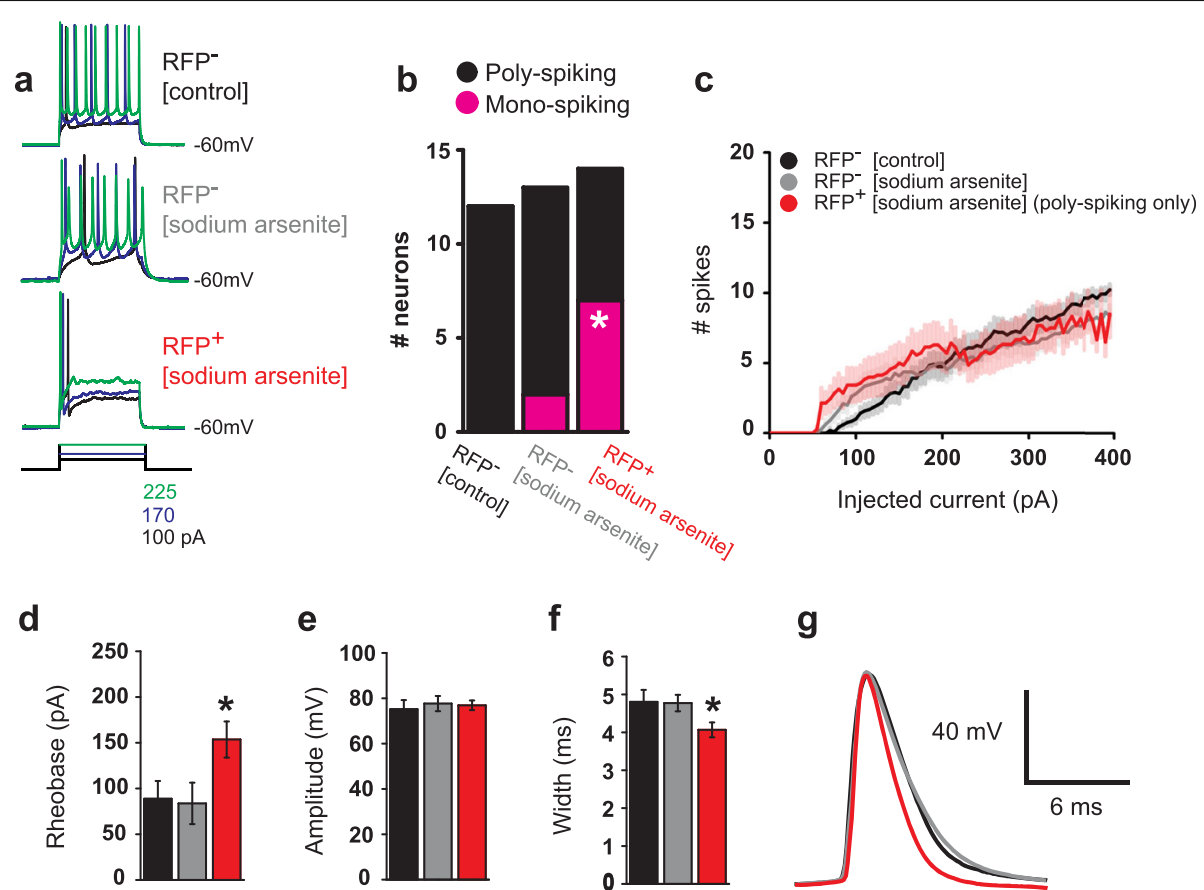

e

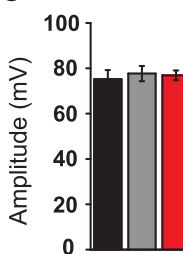

f

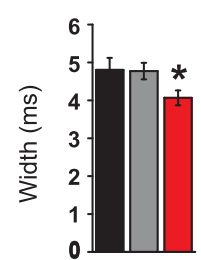

g

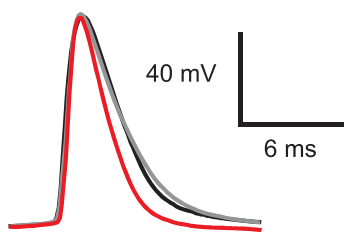

$\mathrm{RFP}^{-}$[control]

RFP- [sodium arsenite]

$\mathrm{RFP}^{+}$[sodium arsenite]

Fig. 5 RFP-positive cortical pyramidal neurons exhibit reduced excitability. a Representative current-clamp traces of action potentials (AP) evoked by current injection at rheobase (black) and above threshold (blue and green). While AP frequency generally increased with step amplitude in RFP $^{-}$neurons from either control- or arsenite-treatment groups, RFP ${ }^{+}$neurons tended to elicit single spikes regardless of stimulus intensity (bottom trace). b Quantification of mono-spiking neurons recorded in each condition. A larger proportion of RFP-expressing neurons were classified as mono-spiking when compared to both RFP-negative groups $\left[X^{2}(1,40)=7.38, P<0.05\right.$ by chi-square test]. c Stimulus-frequency curves show that poly-spiking RFP ${ }^{+}$neurons (red) follow a profile similar to RFP ${ }^{-}$neurons from both arsenite and untreated groups (black and gray) $[F(1,99)=0.34, P=0.71$ by one-way repeated measures ANOVA]. $\mathbf{d}$ The average amount of depolarizing current required to elicit an action potential from rest (rheobase) was increased in $\operatorname{RFP}^{+}$neurons $\left[F(2,30)=3.33, P<0.05\right.$ by one-way ANOVA, ${ }^{*} P<0.05$ by Tukey test]. e No differences were observed in the peak amplitude of 1st APs between groups $[F(2,29)=0.17, P=0.86$ one-way ANOVA]. $\mathbf{f}$ RFP+ neurons elicited narrower 1 st APs when compared to RFP- neurons from both arsenite and untreated groups $\left[F(2,31)=6.13, P<0.005\right.$ by one-way ANOVA, ${ }^{*} P<$ 0.05 by Tukey test]. $\mathbf{g}$ Representative spike traces from individual RFP ${ }^{+}$, RFP ${ }^{-}$, and control neurons demonstrating that 1 st AP width is reduced in RFP $^{+}$neurons, without a change in peak amplitude

\section{Tracing the lineage of cells prenatally affected by environmental insults}

Prenatal exposure to alcohol and arsenic has been shown to cause developmental neurotoxicity, affecting intellectual, emotional, and social functions permanently throughout later life [22, 24-29]. In the reporter transgenic mice, we observed RFP reporter expression, which reflects prior activation of HS signaling, in both neurons and glial cells broadly in the postnatal brain. The affected brain regions include the cerebral cortex and hypothalamus, in which the impact of early exposure to alcohol and arsenic has been suspected to contribute to cognitive and behavioral deficits [31-35].

Our previous studies have shown that HSE drives reporter expression not only in the brain but also in many organs and tissues throughout the body of the embryo, including the eyes, heart, lung, and limb bud, in response to prenatal exposure to environmental insults [12]. Detection of cellular damage is also not limited to that of prenatal insults, but includes postnatal insults such as spinal cord or sciatic nerve injury [36]. Therefore, it is expected that these transgenic mice enable long-term tracing of neural as well as non-neural cell lineages affected by various types of chemical, infectious, or physical environmental factors during both prenatal and postnatal periods. The reporter transgenic mice were generated by crossing the HSE-FLPo mice with the RC::FLTG mice, which can also express enhanced green fluorescent protein (EGFP) in a Cre recombinase-dependent manner. This will help to investigate RFP reporter-positive and -negative cells within EGFP-labeled specific cell populations.

Functional impact of prenatal exposure to environmental insults on cortical neurons

In our study of cortical neurons, we tested for physiological alterations that were specific to neuronal lineages 
that underwent HS activation at their progenitor stage as a result of sodium arsenite exposure. We found that intrinsic cell membrane properties were identical between $\mathrm{RFP}^{+}$and $\mathrm{RFP}^{-}$pyramidal neurons in layers II and III, indicating that HS activation, and the associated impact of arsenite exposure during pregnancy, are unlikely to bestow obvious long-term changes to cortical cell membranes in offspring. Likewise, the membrane properties of $\mathrm{RFP}^{+}$neurons were found to be no different from $\mathrm{RFP}^{-}$neurons recorded in untreated mice, suggesting no obvious effect of prenatal stressor (sodium arsenite) on membrane properties in mature cortical neurons. Additionally, spontaneous excitatory inputs onto $\mathrm{RFP}^{+}$cells appeared to be unchanged compared to $\mathrm{RFP}^{-}$cells from both arsenite-treated and untreated mice, indicating no effect of prenatal HS activation or sodium arsenite exposure on the rate of vesicle release at glutamatergic synapses, or on post-synaptic signaling mechanisms such as glutamate receptor expression/ function.

Despite the numerous consistencies we observed between $\mathrm{RFP}^{+}$and $\mathrm{RFP}^{-}$cortical pyramidal neurons, current-clamp recordings revealed a distinct phenotype of reduced excitability in $\mathrm{RFP}^{+}$cells. When subjected to depolarizing current injection, half of $\mathrm{RFP}^{+}$neurons elicited only a single action potential, rather than a train of spikes, at stimulations well above the firing threshold-a phenomenon that was not observed in control neurons. Interestingly, a similar low-excitability phenotype was reported in lower layer cortical pyramidal neurons as a result of early ethanol exposure, where single-spiking was also prominent [37]. This restricted form of action potential generation, associated with increased rheobase current, narrowed spike width, and no change in resting potential, input resistance, or AP amplitude, can be attributed to low threshold, slowly inactivating, potassium $\left(\mathrm{K}^{+}\right)$currents as described previously [38-42]. In the cortex, such hyperpolarizing $\mathrm{K}^{+}$currents are typically mediated by Kv1.1 subunit-containing voltage-gated $\mathrm{K}^{+}$ channels, which activate near the AP threshold $(\sim-45$ $\mathrm{mV}$ ) and serve to prevent repetitive firing during a prolonged stimulus [43]. In the context of our data, it is possible that Kv1.1 channels have become upregulated following environmental stress, specifically in $\mathrm{RFP}^{+}$neurons, and enforce single-spike responses to membrane depolarization. Future studies might test this hypothesis by assessing the ability of Kv1-selective channel blockers to reverse the low output phenotype observed in $\mathrm{RFP}^{+}$ neurons.

It is well known that arsenic negatively impacts brain development, as rodent studies have reported deficits in neuromotor development $[44,45]$ and cognitive flexibility [24] as consequences of prenatal exposure. Our finding, that excitability is reduced among pyramidal neurons located in layers II and III of the primary motor cortex, provides a possible explanation for arseniteinduced impairments, as it represents a striking loss of output from a cell population that is critical for coordinating sensorimotor responses [46]. Importantly, the observed single-spiking phenotype was specific to neurons that expressed the RFP reporter and had therefore undergone HS activation earlier in their lineage. Thus, our results point to HS signaling-activated neural populations as promising therapeutic targets for the treatment of prenatal arsenic exposure. Other important questions would be whether abnormal characteristics are found commonly in reporter-positive cells in brains exposed to different types of environmental stress, and what behavioral consequences are brought by observed cellular changes. Given our results were obtained under extreme exposure conditions (i.p. injections at high doses for a short period), studies using different, more clinically relevant conditions (e.g., chronic drinking model) would also be necessary.

\section{Mechanisms for the long-term effects of prenatal exposure}

As discussed above, it is likely that the long-term impact of prenatal exposure to environmental insults is mediated by altered regulation of key molecules, such as voltage-gated ion channels, in the mature brain. Defining the pathological molecular profiles in reporter-positive cells would be of particular interest. One possible mechanism for long-term changes in gene regulation is that earlier impacts of exposure and HS activation in neural progenitor cells cause epigenetic modifications that are inherited by mature neurons originated from these progenitor cells. In fact, modification of epigenetic signatures by prenatal exposure to alcohol [47-50] and arsenic [51-53] has been shown and suggested to have permanent consequences on brain development. HS signaling has also been shown to affect epigenetic regulations, causing long-term changes in gene expression [13, 54]. Elucidating the epigenetic mechanisms that may underlie long-term adverse outcomes of prenatal exposure to environmental insults will facilitate our research toward the discovery of novel therapeutic approaches for intellectual disability and other neuropsychiatric disorders. It would be also important to expand similar approaches to other stress response pathways, such as the ER stress response pathway [55, 56], to uncover the whole picture of cellular damage established in utero and propagated throughout life.

\section{Conclusions}

Here, we report the generation and use of new reporter transgenic mice that enable long-term tracing of cell lineages that once have responded to environmental 
insults, via activation of HS signaling. This new transgenic mouse line will serve as a versatile tool to fill the gap in our knowledge on the mechanisms underlying the long-term impacts of environmental insults that often manifest as intellectual disability or other neurodevelopmental disorders long after the actual exposure.

\section{Supplementary Information}

The online version contains supplementary material available at https://doi. org/10.1186/s11689-020-09339-w.

Additional file 1: Supplementary Figure 1. Confirmation of transgene insertion in the founder lines. PCR was performed with two different primer sets using the tail tissue of founder lines (numbers 1-11 for lines A1-A11, respectively) for detection of the transgene (393 bp in upper panel, $415 \mathrm{bp}$ in lower panel). The Rgs7 locus was used as the internal control (507 bp in upper panel, 689 bp in lower panel).

Additional file 2: Supplementary Figure 2. Genomic locus of the transgene integration in chromosome 18 in the founder line A4. (a) Whole genome sequencing with $3 X$ coverage defined an intronic region of Tcerg1 gene locus in chromosome 18 as the region of the transgene insertion in the F2 generation. The direction of the transgene in intronic regions is opposite to the transcriptional direction of the endogenous gene. Each one of the paired reads includes a partial sequence of FLPO. (b) PCR using the primer pair of FLPoF/Chr18R after several generations of breeding (after the F5 generation) resulted in a $1500 \mathrm{bp}$ product (arrow) from the sample of founder line A4, confirming the transgene insertion in the identified locus. No PCR product was amplified from the sample of wild-type (WT) mice. Lane 1-4: DNA ladder, A4 genomic DNA input, A4 PCR product, and WT PCR product, respectively.

Additional file 3: Supplementary Figure 3. Genomic locus of the transgene integration in chromosome 19 in the founder line A4. (a) Whole genome sequencing identified transgene insertion also in the 29 intronic region of Ablim 1 gene in chromosome 19 in the F2 generation of $A 4$. The direction of the transgene is opposite to the transcriptional direction of the endogenous gene. (b) PCR was performed to validate the insertion on the transgene in the founder line A4 using the primer pair of chr19F/Chr19R after several generations of breeding (after the F5 generation). The size of PCR product detected by the BioAnalyzer was $600 \mathrm{bp}$ (arrow), which is expected to be amplified from the intrinsic genomic locus, as shown by the same size of product also amplified from the WT sample. Consistently, the product of $>2000 \mathrm{bp}$, which is expected to be amplified if the transgene is inserted, was not detected. These results indicate that the transgene in the locus in chromosome 19 was not inherited through the generations of breeding in the line A4. Lane 1-3: DNA ladder, A4 PCR product, and WT PCR product, respectively.

Additional file 4: Supplementary Figure 4. Prenatal exposure to sodium arsenite or ethanol does not affect body, brain and liver weights. No differences were observed in the body weight (a), brain weight (b), and liver weight (c) between control, sodium arsenite-exposed, and ethanol-exposed groups at P20 $[F(2,6)=0.51, P=0.62(a), F(2,6)=0.09, P=$ $0.92(b)$, and $F(2,6)=0.24, P=0.79$ (c), all by one-way ANOVA].

\section{Abbreviations}

AP: Action potential; DIC: Differential interference contrast; EGFP: Enhanced green fluorescent protein; ER: Endoplasmic reticulum; EtOH: Ethanol; FASD: Fetal alcohol spectrum disorders; FLPo: Codon-optimized flippase recombinase; FRT: Flippase recombinase-recombination target; HS: Heat shock; HSE: Heat shock response element; i.p.: Intraperitoneal; RFP: Red fluorescent protein; sEPSC: Spontaneous excitatory post-synaptic current; WT: Wild-type

\section{Acknowledgements}

We thank Hannah E. Zikria-Hagemeier and Shivaprasad Bhuvanendran for technical assistance.

\section{Authors' contributions}

P.R., K.H.-T., and M.T. designed the project. S.M., S.J.P., T.S., and Y.I.K. performed experiments, S.M., S.J.P., N.A., Y.I.K., K.H.-T., and M.T. analyzed the data. S.M., S.J.P., P.R., K.H.-T., and M.T. wrote the manuscript. Y.I.K. commented on the manuscript. The authors read and approved the final manuscript.

\section{Funding}

This work was supported by National Institute of Health Grants R01AA025215 (to K.H.-T.), R01AA026272 (to M.T. and K.H.-T.), R01DA023999 (to P.R.), UH2AA026106 as a part of the Collaborative Initiative on Fetal Alcohol Spectrum Disorders (CIFASD)(to K.H.-T. and M.T.), and Scott-Gentle Foundation (to K.H.-T. and M.T.). This study was also supported by Award Number 1U54HD090257-01 from the NIH, District of Columbia Intellectual and Developmental Disabilities Research Center Award (DC-IDDRC) program.

\section{Availability of data and materials}

Whole-genome sequence data is available in SRA (SAMN16895777). The reporter transgenic mice will be made available from The Jackson Laboratory.

Ethics approval and consent to participate

All procedures were performed in accordance with and approved by the Children's National Hospital Institutional Animal Care and Use Committee.

\section{Consent for publication}

Not applicable.

\section{Competing interests}

The authors declare that they have no competing interests.

\section{Author details}

${ }^{1}$ Center for Neuroscience Research, Children's Research Institute, Children's National Hospital, Washington, DC, USA. ${ }^{2}$ Department of Obstetrics and Gynecology, Tokyo Medical University, Tokyo, Japan. ${ }^{3}$ Institute of Biomedical Sciences, School of Medicine and Health Sciences, The George Washington University, Washington, DC, USA. ${ }^{4}$ Department of Neuroscience and Kavli Institute for Neuroscience, Yale University, New Haven, CT, USA. ${ }^{5}$ Department of Pharmacology, Pennsylvania State University College of Medicine, Hershey, PA, USA. 'Department of Biochemistry and Molecular Biology, Institute for Personalized Medicine, Pennsylvania State University College of Medicine, Hershey, PA, USA. 'Department of Pediatrics, Pharmacology and Physiology, School of Medicine and Health Sciences, The George Washington University, Washington, DC, USA.

Received: 23 October 2019 Accepted: 13 November 2020

Published online: 16 December 2020

\section{References}

1. Kleiber ML, Laufer Bl, Wright E, Diehl EJ, Singh SM. Long-term alterations to the brain transcriptome in a maternal voluntary consumption model of fetal alcohol spectrum disorders. Brain Res. 2012;1458:18-33.

2. Caldwell KK, Sheema S, Paz RD, Samudio-Ruiz SL, Laughlin MH, Spence NE, et al. Fetal alcohol spectrum disorder-associated depression: evidence for reductions in the levels of brain-derived neurotrophic factor in a mouse model. Pharmacol Biochem Behav. 2008;90(4):614-24.

3. Cheema ZF, West JR, Miranda RC. Ethanol induces Fas/Apo [apoptosis]-1 mRNA and cell suicide in the developing cerebral cortex. Alcohol Clin Exp Res. 2000;24(4):535-43

4. McAlhany RE Jr, West JR, Miranda RC. Glial-derived neurotrophic factor (GDNF) prevents ethanol-induced apoptosis and JUN kinase phosphorylation. Brain Res Dev Brain Res. 2000;119(2):209-16.

5. Ramanathan R, Wilkemeyer MF, Mittal B, Perides G, Charness ME. Alcohol inhibits cell-cell adhesion mediated by human L1. J Cell Biol. 1996;133(2): 381-90.

6. Ishii S, Hashimoto-Torii K. Impact of prenatal environmental stress on cortical development. Front Cell Neurosci. 2015;9:207.

7. Schmitt A, Malchow B, Hasan A, Falkai P. The impact of environmental factors in severe psychiatric disorders. Front Neurosci. 2014;8:19.

8. Hashimoto-Torii K, Kawasawa Yl, Kuhn A, Rakic P. Combined transcriptome analysis of fetal human and mouse cerebral cortex exposed to alcohol. Proc Natl Acad Sci U S A. 2011;108(10):4212-7. 
9. Hashimoto-Torii K, Torii M, Fujimoto M, Nakai A, El Fatimy R, Mezger V, et al. Roles of heat shock factor 1 in neuronal response to fetal environmental risks and its relevance to brain disorders. Neuron. 2014;82(3):560-72.

10. Ishii S, Hashimoto-Torii KHSF. Modulates neural development under Normal and stress conditions. Japan: Springer; 2016.

11. Ishii S, Torii M, Son Al, Rajendraprasad M, Morozov YM, Kawasawa Yl, et al. Variations in brain defects result from cellular mosaicism in the activation of heat shock signalling. Nat Commun. 2017;8:15157.

12. Torii M, Sasaki M, Chang YW, Ishii S, Waxman SG, Kocsis JD, et al. Detection of vulnerable neurons damaged by environmental insults in utero. Proc Natl Acad Sci U S A. 2017;114 (9):2367-72.

13. Lamke J, Brzezinka K, Altmann S, Baurle I. A hit-and-run heat shock factor governs sustained histone methylation and transcriptional stress memory. EMBO J. 2016;35(2):162-75

14. Raymond CS, Soriano P. High-efficiency FLP and PhiC31 site-specific recombination in mammalian cells. PLoS One. 2007;2(1):e162.

15. Ming R, Hou S, Feng Y, Yu Q, Dionne-Laporte A, Saw JH, et al. The draft genome of the transgenic tropical fruit tree papaya (Carica papaya Linnaeus). Nature. 2008;452(7190):991-6.

16. Langmead B, Salzberg SL. Fast gapped-read alignment with bowtie 2. Nat Methods. 2012:9(4):357-9.

17. Ji Y, Abrams N, Zhu W, Salinas E, Yu Z, Palmer DC, et al. Identification of the genomic insertion site of Pmel-1 TCR alpha and beta transgenes by nextgeneration sequencing. PLoS One. 2014;9(5):e96650.

18. Lambirth KC, Whaley AM, Schlueter JA, Bost KL, Piller KJ. CONTRAILS: a tool for rapid identification of transgene integration sites in complex, repetitive genomes using low-coverage paired-end sequencing. Genomics Data. 2015; 6:175-81.

19. Li H, Durbin R. Fast and accurate short read alignment with burrowswheeler transform. Bioinformatics. 2009;25(14):1754-60.

20. Mohammad S, Page SJ, Wang L, Ishii S, Li P, Sasaki T, et al. Kenn2 blockade reverses learning deficits in a mouse model of fetal alcohol spectrum disorders. Nat Neurosci. 2020;23(4):533-43.

21. Suzuki T, Tsurusaki Y, Nakashima M, Miyake N, Saitsu H, Takeda S, et al. Precise detection of chromosomal translocation or inversion breakpoints by whole-genome sequencing. J Hum Genet. 2014;59(12):649-54.

22. Mattson SN, Riley EP. A review of the neurobehavioral deficits in children with fetal alcohol syndrome or prenatal exposure to alcohol. Alcohol Clin Exp Res. 1998;22(2):279-94

23. Clarke ME, Gibbard WB. Overview of fetal alcohol spectrum disorders for mental health professionals. Can Child Adolesc Psychiatr Rev. 2003; 12(3):57-63.

24. Aung KH, Kyi-Tha-Thu C, Sano K, Nakamura K, Tanoue A, Nohara K, et al. Prenatal exposure to arsenic impairs behavioral flexibility and cortical structure in mice. Front Neurosci. 2016;10:137.

25. Caldwell KE, Labrecque MT, Solomon BR, Ali A, Allan AM. Prenatal arsenic exposure alters the programming of the glucocorticoid signaling system during embryonic development. Neurotoxicol Teratol. 2015;47:66-79.

26. Martinez EJ, Kolb BL, Bell A, Savage DD, Allan AM. Moderate perinatal arsenic exposure alters neuroendocrine markers associated with depression and increases depressive-like behaviors in adult mouse offspring. Neurotoxicology. 2008;29(4):647-55.

27. Rodriguez VM, Carrizales L, Mendoza MS, Fajardo OR, Giordano M. Effects of sodium arsenite exposure on development and behavior in the rat. Neurotoxicol Teratol. 2002;24(6):743-50.

28. Tyler CR, Allan AM. The effects of arsenic exposure on neurological and cognitive dysfunction in human and rodent studies: a review. Curr Environ Health Rep. 2014;1:132-47.

29. Htway SM, Sein MT, Nohara K, Win-Shwe TT. Effects of developmental arsenic exposure on the social behavior and related gene expression in $\mathrm{C} 3 \mathrm{H}$ adult male mice. Int J Environ Res Public Health. 2019;16(2):174.

30. Pignataro L. Alcohol protects the CNS by activating HSF1 and inducing the heat shock proteins. Neurosci Lett. 2019;713:134507.

31. Bekdash R, Zhang C, Sarkar D. Fetal alcohol programming of hypothalamic proopiomelanocortin system by epigenetic mechanisms and later life vulnerability to stress. Alcohol Clin Exp Res. 2014;38(9):2323-30.

32. Hwang HM, Ku RY, Hashimoto-Torii K. Prenatal environment that affects neuronal migration. Front Cell Dev Biol. 2019;7:138.

33. Olney JW, Wozniak DF, Jevtovic-Todorovic V, Farber NB, Bittigau P, Ikonomidou C. Drug-induced apoptotic neurodegeneration in the developing brain. Brain Pathol. 2002;12(4):488-98.
34. Tolins M, Ruchirawat M, Landrigan P. The developmental neurotoxicity of arsenic: cognitive and behavioral consequences of early life exposure. Ann Glob Health. 2014;80(4):303-14.

35. Weinberg J, Sliwowska JH, Lan N, Hellemans KG. Prenatal alcohol exposure: foetal programming, the hypothalamic-pituitary-adrenal axis and sex differences in outcome. J Neuroendocrinol. 2008;20(4):470-88.

36. Hashimoto-Torii K, Sasaki M, Chang YW, Hwang H, Waxman SG, Kocsis JD, et al. Detection of local and remote cellular damage caused by spinal cord and peripheral nerve injury using a heat shock signaling reporter system. IBRO Rep. 2018:5:91-8.

37. Granato A, Palmer LM, De Giorgio A, Tavian D, Larkum ME. Early exposure to alcohol leads to permanent impairment of dendritic excitability in neocortical pyramidal neurons. J Neurosci. 2012;32(4):1377-82.

38. Brew HM, Forsythe ID. Two voltage-dependent $\mathrm{K}+$ conductances with complementary functions in postsynaptic integration at a central auditory synapse. J Neurosci. 1995;15(12):8011-22.

39. Glazebrook PA, Ramirez AN, Schild JH, Shieh CC, Doan T, Wible BA, et al. Potassium channels Kv1.1, Kv1.2 and Kv1.6 influence excitability of rat visceral sensory neurons. J Physiol. 2002;541(Pt 2):467-82.

40. Karcz A, Rubsamen R, Kopp-Scheinpflug C. Low-threshold potassium currents stabilize IID-sensitivity in the inferior colliculus. Front Neural Circuits. 2012;6:60

41. Ovsepian SV, LeBerre M, Steuber V, O'Leary VB, Leibold C, Oliver Dolly J. Distinctive role of KV1.1 subunit in the biology and functions of low threshold $\mathrm{K}(+)$ channels with implications for neurological disease. Pharmacol Ther. 2016;159:93-101.

42. Watanabe T, Shimazaki T, Oda Y. Coordinated expression of two types of low-threshold $\mathrm{K}(+)$ channels establishes unique single spiking of mauthner cells among segmentally homologous neurons in the zebrafish hindbrain. eNeuro. 2017:4(5):0249-17.

43. Guan D, Lee JC, Higgs MH, Spain WJ, Foehring RC. Functional roles of Kv1 channels in neocortical pyramidal neurons. J Neurophysiol. 2007; 97(3):1931-40.

44. Colomina MTA, L M, Domingo JL, Corbella J. Influence of maternal restraint stress on arsenic-induced pre- and postnatal alterations in mice. Psychobiology. 1996;24(3):227-34.

45. Markowski VP, Reeve EA, Onos K, Assadollahzadeh M, McKay N. Effects of prenatal exposure to sodium arsenite on motor and food-motivated behaviors from birth to adulthood in C57BL6/J mice. Neurotoxicol Teratol. 2012:34(2):221-31.

46. Huber D, Gutnisky DA, Peron S, O'Connor DH, Wiegert JS, Tian L, et al. Multiple dynamic representations in the motor cortex during sensorimotor learning. Nature. 2012;484(7395):473-8.

47. Hicks SD, Middleton FA, Miller MW. Ethanol-induced methylation of cell cycle genes in neural stem cells. J Neurochem. 2010;1 14(6):1767-80.

48. Kaminen-Ahola N, Ahola A, Maga M, Mallitt KA, Fahey P, Cox TC, et al. Maternal ethanol consumption alters the epigenotype and the phenotype of offspring in a mouse model. PLoS Genet. 2010;6(1):e1000811.

49. Khalid O, Kim JJ, Kim HS, Hoang M, Tu TG, Elie O, et al. Gene expression signatures affected by alcohol-induced DNA methylomic deregulation in human embryonic stem cells. Stem Cell Res. 2014;12(3):791-806.

50. Kleiber ML, Diehl EJ, Laufer BI, Mantha K, Chokroborty-Hoque A, Alberry B, et al. Long-term genomic and epigenomic dysregulation as a consequence of prenatal alcohol exposure: a model for fetal alcohol spectrum disorders. Front Genet. 2014:5:161.

51. Tyler CR, Hafez AK, Solomon ER, Allan AM. Developmental exposure to 50 parts-per-billion arsenic influences histone modifications and associated epigenetic machinery in a region- and sex-specific manner in the adult mouse brain. Toxicol Appl Pharmacol. 2015;288(1):40-51.

52. Tyler CR, Labrecque MT, Solomon ER, Guo X, Allan AM. Prenatal arsenic exposure alters REST/NRSF and microRNA regulators of embryonic neural stem cell fate in a sex-dependent manner. Neurotoxicol Teratol. 2017:59:1-15.

53. Rager JE, Auerbach SS, Chappell GA, Martin E, Thompson CM, Fry RC. Benchmark dose modeling estimates of the concentrations of inorganic arsenic that induce changes to the neonatal transcriptome, proteome, and epigenome in a pregnancy cohort. Chem Res Toxicol. 2017;30(10):1911-20.

54. Miozzo F, Arnould H, de Thonel A, Schang AL, Saberan-Djoneidi D, Baudry A, et al. Alcohol exposure promotes DNA methyltransferase DNMT3A upregulation through reactive oxygen species-dependent mechanisms. Cell Stress Chaperones. 2018;23(1):115-26. 
55. Iwawaki T, Akai R, Kohno K, Miura M. A transgenic mouse model for monitoring endoplasmic reticulum stress. Nat Med. 2004;10(1): 98-102.

56. Mao C, Dong D, Little E, Luo S, Lee AS. Transgenic mouse model for monitoring endoplasmic reticulum stress in vivo. Nat Med. 2004;10(10): 1013-4 author reply 4.

\section{Publisher's Note}

Springer Nature remains neutral with regard to jurisdictional claims in published maps and institutional affiliations.

Ready to submit your research? Choose BMC and benefit from:

- fast, convenient online submission

- thorough peer review by experienced researchers in your field

- rapid publication on acceptance

- support for research data, including large and complex data types

- gold Open Access which fosters wider collaboration and increased citations

- maximum visibility for your research: over $100 \mathrm{M}$ website views per year

At BMC, research is always in progress.

Learn more biomedcentral.com/submissions 